\title{
Broad as a barn
}

\begin{abstract}
If you were ever puzzled about the fact that the detectors at the Large Hadron Collider record huge datasets despite the tiny probability of two protons colliding, this is for you. Steven Goldfarb and Katarina Anthony connect the dots.
\end{abstract}

$\mathrm{C}=$ hances are, in four billion years, when the Milky Way collides with Andromeda, our Sun will be just fine. In fact, most likely, none of the hundreds of billions of stars in either galaxy will come into contact ${ }^{1}$. Despite appearing to be densely packed when viewed from afar, there is still a lot of space between the stars of both galaxies.

This is no surprise to the scientists working on the Large Hadron Collider (LHC) at CERN ${ }^{2}$. Here, similar collisions are produced 40 million times a second, as tiny bunches containing 100 billion protons each are slammed into each other in the centre of the detectors. Only by using powerful, superconducting magnets are LHC scientists and engineers able to coax roughly 50 pairs of protons to collide in the ATLAS and CMS experiments. Inner space, it seems, is also quite sparse.

But how do scientists determine the likelihood of two objects, as small as protons or as big as stars, colliding? Essential to this is the measurement of a system's crosssection, which is defined as its area in the plane transverse to its direction of flight. The larger the cross-section, the higher the probability of collision. That is, big stars are more likely to run into each other than small stars. Our own star has a cross-section of about 1.5 trillion square kilometres (which is about medium size in this galaxy).

Unlike macroscopic objects, particles do not have well-defined physical surfaces. Thus, in high-energy physics, the unit of barn (b) is used to express particle crosssections, which represents the area within which the particles will interact. One barn is equal to $10^{-24} \mathrm{~cm}^{2}$ - roughly the crosssection of a uranium nucleus. Its name originates from the Second World War when scientists working on the Manhattan Project considered that colliding such 'large' atoms to be as easy as 'hitting the side of a barn'.

Of course, you might wonder how many barn would fit on the side of an actual barn. Let's assume that the side of a typical barn is about $10-15 \mathrm{~m}$ long by $8-10 \mathrm{~m}$ tall, giving it a surface area of about $100 \mathrm{~m}^{2}$ or $10^{6} \mathrm{~cm}^{2}$. That's roughly $10^{30}$ barn on one side of a barn.

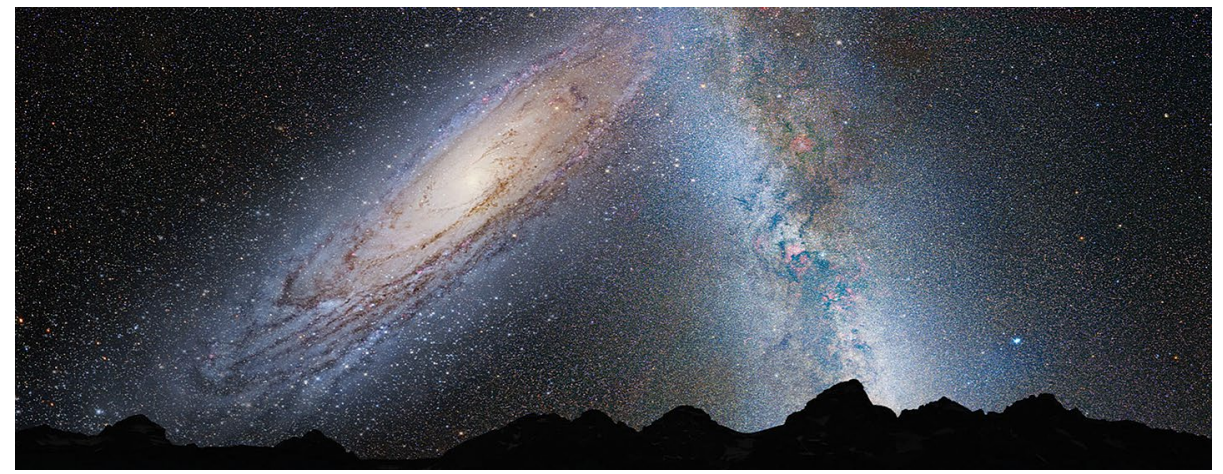

Credit: NASA; ESA; Z. Levay and R. van der Marel, STScl; T. Hallas; and A. Mellinger

Interestingly, for particle colliders, the interactions and thus the cross-sections depend on the energy of the beams. For LHC operation at $13 \times 10^{12} \mathrm{eV}(\mathrm{TeV})$, the total inelastic cross-section of the colliding protons has been measured to be around $78 \times 10^{-27} \mathrm{~cm}^{2}$ or 78 millibarn (mb) (ref. ${ }^{4}$ ). Other units commonly used in particle physics extend over several orders of magnitude: the microbarn $(\mu \mathrm{b})=10^{-30} \mathrm{~cm}^{2}$, nanobarn $(\mathrm{nb})=10^{-33} \mathrm{~cm}^{2}$, picobarn $(\mathrm{pb})=$ $10^{-36} \mathrm{~cm}^{2}$ and femtobarn $(\mathrm{fb})=10^{-39} \mathrm{~cm}^{2}$.

Just for fun, some have referred to the microbarn as an outhouse, but you'll probably never find that in a peer-reviewed publication.

You will also find in publications numbers expressed in inverse barns or $1 / b=b^{-1}$. That's because physicists express the number of particle collisions per unit time in terms of the cross-section. At the LHC, this rate, called the instantaneous luminosity, reached a peak in 2018 of around $20 \mathrm{nb}^{-1} \mathrm{~s}^{-1}$. Adding up all the collisions produced in the ATLAS experiment, for example, over that year gives an integrated luminosity of around $65 \mathrm{fb}^{-1}$ (ref. ${ }^{5}$ ). That represents around $5 \times 10^{15}$ (5 quadrillion) inelastic proton-proton collisions in a single year - a record amount, even for the LHC.

Why the need for so many collisions? At a proton beam energy of $6.5 \mathrm{TeV}$, as was used for the proton-proton collisions during the second run of the LHC from 2015 to 2018, 1 Higgs boson is produced per 2 billion collisions. That means that the $65 \mathrm{fb}^{-1}$ of data recorded by the ATLAS experiment in 2018 yielded around 3 million Higgs bosons. Unfortunately, only 1 in 1,000 of the Higgs decays is detected. Some Higgs decays occur rarely, such as the $0.02 \%$ that decay to muon pairs, and some Higgs decays are just difficult to pick out because they, for example, are very hard to separate from background processes - as is the case for the $60 \%$ decaying into b-quark pairs ${ }^{6}$. So, although measuring rare decays can be like picking a needle out of a haystack, one can fit a lot of hay in a barn, and the LHC is a very big barn, indeed.

Steven Goldfarb ${ }^{1 \star}$ and Katarina Anthony ${ }^{2 \star}$ ${ }^{1}$ University of Melbourne, Melbourne, Victoria, Australia. ${ }^{2}$ Universita degli Studi di Udine, Udine, Italy. *e-mail: steven.goldfarb@cern.ch; katarina.anthony@cern.ch

Published online: 1 April 2019 https://doi.org/10.1038/s41567-019-0490-z

References

1. NASA's Hubble shows Milky Way is destined for head-on collision. https://go.nature.com/2ulxhQH (NASA, 31 May 2012).

2. The Large Hadron Collider at CERN; https://go.nature.com/2u7v7it 3. Perricone, M. Hitting the broad side of a (classified) barn. Symmetry https://go.nature.com/2TJbg7S (1 February 2006).

4. Aaboud, M. et al. (ATLAS Collaboration) Phys. Rev. Lett. 117, 182002 (2016).

5. ATLAS experiment - luminosity public results Run $2 \mathrm{https://}$ go.nature.com/2TIgEYP (accessed 11 March 2019).

6. SM Higgs branching ratios and total decay widths (update in CERN Report4 2016) https://go.nature.com/2Hs7hWF (accessed 11 March 2019).

$\mathrm{m} e \mathrm{~s} \mathrm{\textrm {u }} R \mathrm{E}_{\mathrm{h}}$

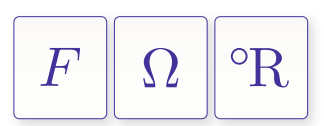

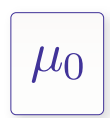

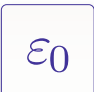

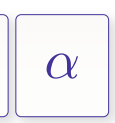

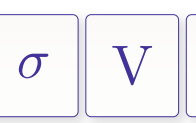

$\mathrm{R}$ 